\title{
Planetary Gear Modeling Using the Power-Oriented Graphs Technique.
}

\author{
Roberto Zanasi, Davide Tebaldi
}

\begin{abstract}
In this paper, the Power-Oriented Graphs (POG) technique is used to model Planetary Gear transmission systems. The full elastic dynamic model of the system is obtained using a fast and direct method which can be easily applied to any type of planetary gear. The rigid and reduced dynamic model of the system when the stiffness coefficients go to infinity is then obtained using a POG congruent state space transformation allowing the user to select which angular speeds are to be maintained in the reduced model. Another interesting aspect of the presented method is that the obtained reduced model is still able to provide the time behaviors of the tangential forces present between each couple of gears of the considered planetary gear system. The presented fast and direct method is then applied to two practical case studies, and simulative results in Matlab/Simulink showing the effectiveness of the method are finally reported and commented.
\end{abstract}

\section{INTRODUCTION}

Planetary gear transmission systems are amply used in several applications, including the design of new hybrid architectures in the automotive and agricultural fields, where such elements play a fundamental role as power-split devices. The need of providing direct and accurate dynamic planetary gear models is therefore becoming more and more important, in order to investigate the system behavior in all its aspects. In [1], the authors mainly focus on the design part of planetary gear systems. In [2], the authors propose the usage of the lumped parameter dynamic model for modeling planetary gears; whereas in [3], the authors model the rigid gear subsystem, composed of a sun gear, the planet gears and a planet carrier, by using a lumped parameter model, and the elastic ring gear subsystem by using a finite element model. The problem of the dynamic modeling of a planetary gear system is also addressed by the authors in [4]. In this latter paper, the authors focus on the modeling of a specific type of planetary gear by using the classical state-space approach. An automated approach for modeling planetary gears is proposed by the authors in [5]. In this study, the classical state-space approach is once again adopted.

In this paper, we propose a direct and systematic method to model planetary gear systems using the Power-Oriented Graphs technique, see [6]. The proposed method makes it possible to model any type of planetary gear system by also considering the translational springs being present among the gears. In addition, the user-friendly graphical representation of the POG modeling technique provides the user with a quick understanding of the system dynamic equations. The use of the POG state-space model rather than the classical

Roberto Zanasi and Davide Tebaldi are with the Department of Engineering "Enzo Ferrari", University of Modena and Reggio Emilia, Modena, Italy, e-mail: roberto.zanasi@unimore.it, davide.tebaldi@unimore.it
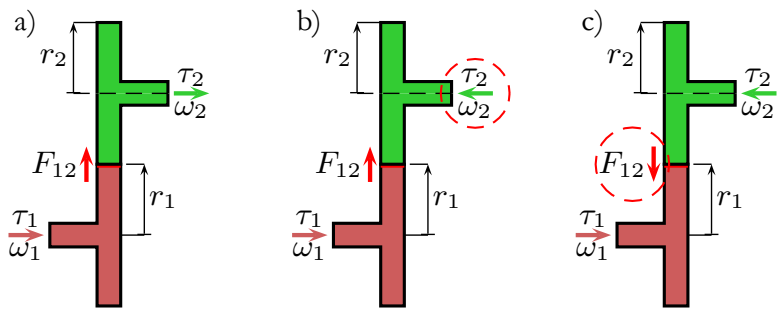

Fig. 1. Gear transmission: positive directions of the system variables.

state-space approach brings several advantages, among which we can mention the potentiality of applying a congruent state-space transformation allowing to reduce the order of the system. Indeed, the study presented in this paper shows that, when the stiffness coefficients go to infinity, the reduced rigid model can be obtained by using a POG congruent state space transformation. From the reduced rigid model, it is also possible to obtain the tangential forces associated with the elastic elements of the full elastic model.

The paper is organized as follows: In Sec. II, we focus on the modeling of a gear transmission. In Sec. III, we generalize the rules presented in Sec. II in order to describe the systematic approach for modeling planetary gears allowing to compute the radius matrix, which fully defines the planetary gear under consideration. Additionally, we apply the presented systematic modeling approach to a one-stage planetary gear. In Sec. IV, we focus on a more complex case study: the modeling of a two-stages planetary gear. Finally, Sec. V illustrates the conclusions of the work presented in this paper.

\section{MODELING A GEAR TRANSMISSION}

Let us consider the gear transmission shown in Fig. 1 case a). The figure highlights the positive directions of the system variables: the angular velocities of the two gears, $\omega_{1}$ and $\omega_{2}$, together with the tangential force $F_{12}$ that gear 1 transmits to gear 2. The POG block scheme of the gear transmission in Fig. 1 case a) is shown in Fig. 2 and the corresponding POG state space equations are reported in (1).

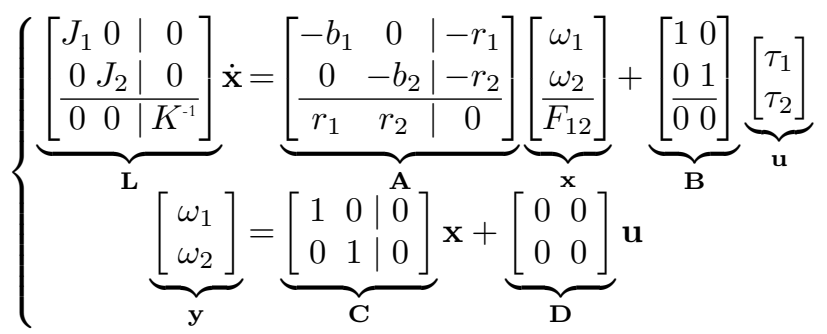

The meaning of the parameters within system (1) is the 


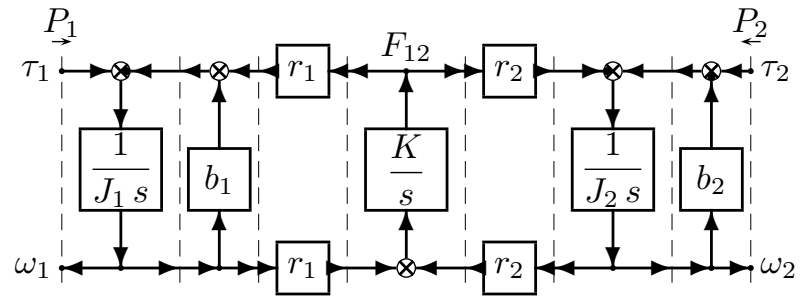

Fig. 2. POG block scheme of a considered gear transmission.

following: $J_{1}, b_{1}$ and $r_{1}$ are the moment of inertia, the linear friction coefficient and the radius of the first gear. Equivalently, parameters $J_{2}, b_{2}$ and $r_{2}$ are the moment of inertia, the linear friction coefficient and the radius of the second gear, whereas parameter $K$ is the stiffness of the contact spring between the first and the second gear. The signs of the parameters within system (1) and Fig. 2 are a direct consequence of the choices about the positive direction of the system state variables $\omega_{1}, \omega_{2}$ and $F_{12}$. Powers $P_{1}=$ $\omega_{1} \tau_{1}$ and $P_{2}=\omega_{2} \tau_{2}$ flowing through the input and output power sections are positive, see Fig. 2, if they are entering the system, meaning that the positive directions of the input torques $\tau_{1}$ and $\tau_{2}$ have been chosen to be equal to the positive directions of the angular velocities $\omega_{1}$ and $\omega_{2}$. One can easily prove that, if the positive direction of variables $\omega_{2}$ and $\tau_{2}$ is reversed, see Fig. 1 case b), the state space equations remain the same except for matrix $\mathbf{A}$, which turns into:

$$
\mathbf{A}_{1}=\left[\begin{array}{cc|c}
-b_{1} & 0 & -r_{1} \\
0 & -b_{2} & r_{2} \\
\hline r_{1} & -r_{2} & 0
\end{array}\right] .
$$

In this case, only the sign of parameter $r_{2}$ is changed. Moreover, by starting from case b) and reversing the positive direction of variable $F_{12}$, see Fig. 1 case c), the state space equations (1) remain unchanged once again except for matrix A, which now turns into:

$$
\mathbf{A}_{2}=\left[\begin{array}{cc|c}
-b_{1} & 0 & r_{1} \\
0 & -b_{2} & -r_{2} \\
\hline-r_{1} & r_{2} & 0
\end{array}\right]
$$

In this case, the signs of both parameters $r_{1}$ and $r_{2}$ within matrix $\mathbf{A}_{2}$ are changed with respect to matrix $\mathbf{A}_{1}$. By rewriting matrix $\mathbf{A}$ in the following form:

$$
\mathbf{A}=\left[\begin{array}{cc}
-\mathbf{B}_{J} & \mathbf{R}^{\mathrm{T}} \\
\mathbf{R} & 0
\end{array}\right], \quad \mathbf{B}_{J}=\left[\begin{array}{cc}
b_{1} & 0 \\
0 & b_{2}
\end{array}\right], \quad \mathbf{R}=\left[r_{1} r_{2}\right]
$$

and focusing on the previous two cases, one can state that: 1) matrix $\mathbf{B}_{J}$ does not change even if the system variables $\omega_{1}$, $\omega_{2}$ and $F_{12}$ change the signs of their positive directions; 2) if the angular velocity $\omega_{i}$ changes the sign of its positive direction, then all the coefficients of the $i$-th column of matrix $\mathbf{R}$ change their signs; 3) if the tangential force $F_{12}$ changes the sign of its positive direction, then all the coefficients of matrix $\mathbf{R}$ change their signs.

\section{Modeling a ONE-STAGe Planetary Gear}

Reference is made to the planetary gear shown in Fig. 3. Within the figure, each gear is characterized by a one-digit

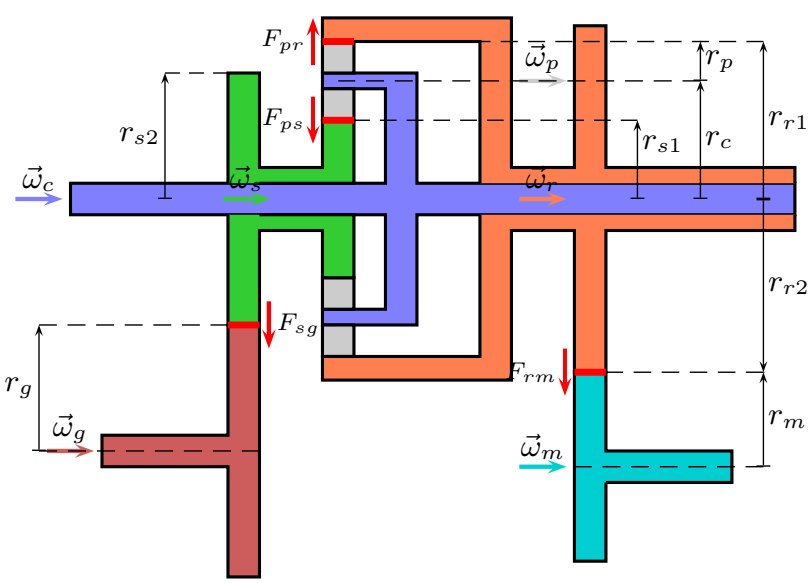

Fig. 3. Basic structure of the considered one-stage planetary gear.

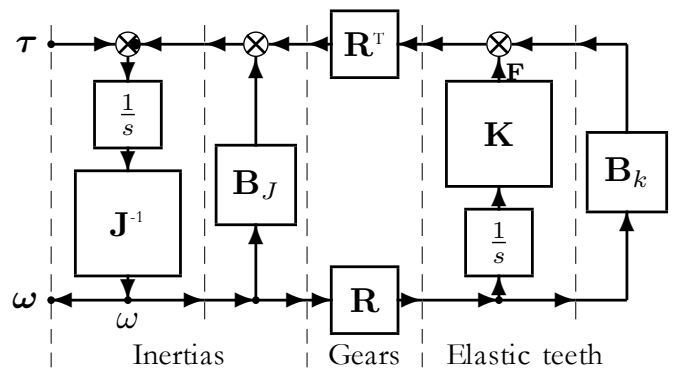

Fig. 4. POG scheme of the considered planetary gear system.

subscript " $i$ ", where $i \in\{c, p, s, r, g, m\}$, and a specific color. Each gear rotates around a horizontal axis, denoted in the figure by a short arrow (i.e. “ $\Longrightarrow$ "), having the same color of the considered gear. The direction of the short arrow indicates the positive direction of the associated angular velocity $\vec{\omega}_{i}$ of gear " $i$ ". The one-digit string " $i$ " also denotes all the parameters and variables associated with gear " $i$ ": $J_{i}$ is the moment of inertia, $b_{i}$ is the linear friction coefficient and $\tau_{i}$ is the input torque applied to the gear.

The gears interact each other by means of their teeth. The contact points of the gears are denoted in Fig. 3 by short red lines (i.e. "- ") representing the stiffness of the teeth where the gears interact each other. The tangential forces $F_{i j}$ transmitted by the contact points are denoted in Fig. 3 by short red vertical arrows (i.e. “ $\downarrow$ ”) representing the positive directions of the contact forces themselves. The twodigits subscript " $i j$ " of the tangential force $F_{i j}$ indicates that such force is positive if oriented from gear " $i$ " to gear " $j$ " and that the spring connecting the two gears has its first terminal connected to gear " $i$ " and its second terminal connected to gear " $j$ ". Such two-digits string also denotes all the parameters and variables associated with the spring " $i j$ ": $K_{i j}$ is the spring stiffness and $b_{i j}$ is the spring linear friction coefficient.

The dynamic equations of the considered system are graphically represented by the POG scheme shown in Fig. 4. The corresponding state space equations are:

$$
\left\{\begin{array}{rl}
\mathbf{L} \dot{\mathbf{x}} & =\mathbf{A} \mathbf{x}+\mathbf{B} \mathbf{u} \\
\mathbf{y} & =\mathbf{B}^{\mathrm{T}} \mathbf{x}
\end{array}, \quad \mathbf{x}=\left[\begin{array}{l}
\boldsymbol{\omega} \\
\mathbf{F}
\end{array}\right], \quad \mathbf{B}=\left[\begin{array}{l}
\mathbf{I} \\
\mathbf{0}
\end{array}\right]\right.
$$


where $\mathbf{x}, \mathbf{u}=\boldsymbol{\tau}, \mathbf{y}=\boldsymbol{\omega}$ are the state, input and output vectors of the system, respectively, $\mathbf{B}$ is the input matrix and $\mathbf{I}$ is an identity matrix of proper dimension. The energy and power matrices $\mathbf{L}$ and $\mathbf{A}$ have the following structure:

$$
\mathbf{L}=\left[\begin{array}{cc}
\mathbf{J} & \mathbf{0} \\
\mathbf{0} & \mathbf{K}^{-1}
\end{array}\right], \quad \mathbf{A}=\left[\begin{array}{cc}
-\mathbf{B}_{J}-\mathbf{R}^{\mathrm{T}} \mathbf{B}_{k} \mathbf{R} & -\mathbf{R}^{\mathrm{T}} \\
\mathbf{R} & \mathbf{0}
\end{array}\right]
$$

The velocity vector $\boldsymbol{\omega}$, the input torque vector $\boldsymbol{\tau}$ and the force vector $\mathbf{F}$ are defined as follows:

$$
\boldsymbol{\omega}=\left[\begin{array}{c}
\omega_{c} \\
\omega_{p} \\
\omega_{s} \\
\omega_{r} \\
\omega_{g} \\
\omega_{m}
\end{array}\right], \quad \boldsymbol{\tau}=\left[\begin{array}{c}
\tau_{c} \\
\tau_{p} \\
\tau_{s} \\
\tau_{r} \\
\tau_{g} \\
\tau_{m}
\end{array}\right], \quad \mathbf{F}=\left[\begin{array}{c}
F_{p s} \\
F_{p r} \\
F_{s g} \\
F_{r m}
\end{array}\right]
$$

The inertia matrix $\mathbf{J}$ and the inertia friction matrix $\mathbf{B}_{J}$ are:

$$
\mathbf{J}=\left[\begin{array}{cccccc}
J_{c} & 0 & 0 & 0 & 0 & 0 \\
0 & J_{p} & 0 & 0 & 0 & 0 \\
0 & 0 & J_{s} & 0 & 0 & 0 \\
0 & 0 & 0 & J_{r} & 0 & 0 \\
0 & 0 & 0 & 0 & J_{g} & 0 \\
0 & 0 & 0 & 0 & 0 & J_{m}
\end{array}\right], \quad \mathbf{B}_{J}=\left[\begin{array}{cccccc}
b_{c} & 0 & 0 & 0 & 0 & 0 \\
0 & b_{p} & 0 & 0 & 0 & 0 \\
0 & 0 & b_{s} & 0 & 0 & 0 \\
0 & 0 & 0 & b_{r} & 0 & 0 \\
0 & 0 & 0 & 0 & b_{g} & 0 \\
0 & 0 & 0 & 0 & 0 & b_{m}
\end{array}\right]
$$

The stiffness matrices $\mathbf{K}$ and $\mathbf{B}_{K}$ are defined as follows:

$$
\mathbf{K}=\left[\begin{array}{cccc}
K_{p s} & 0 & 0 & 0 \\
0 & K_{p r} & 0 & 0 \\
0 & 0 & K_{s g} & 0 \\
0 & 0 & 0 & K_{r m}
\end{array}\right], \quad \mathbf{B}_{K}=\left[\begin{array}{cccc}
b_{p s} & 0 & 0 & 0 \\
0 & b_{p r} & 0 & 0 \\
0 & 0 & b_{s g} & 0 \\
0 & 0 & 0 & b_{r m}
\end{array}\right]
$$

The position of the angular velocities $\omega_{i}$ within vector $\boldsymbol{\omega}$ and of the tangential forces $F_{i j}$ within vector $\mathbf{F}$, see (4), completely and uniquely defines the position of parameters $J_{i}, b_{i}, K_{i j}$ and $b_{i j}$ within diagonal matrices $\mathbf{J}, \mathbf{B}_{J}, \mathbf{K}$ and $\mathbf{B}_{K}$ respectively. The only matrix in Fig. 3 and in the state space equations (2) that fully defines the internal structure of the given planetary gear is the radius matrix $\mathbf{R}$. As far as the considered system is concerned, matrix $\mathbf{R}$ is defined as:

$$
\mathbf{R}=\left[\begin{array}{cccccc}
r_{c} & -r_{p} & -r_{s 1} & 0 & 0 & 0 \\
r_{c} & r_{p} & 0 & -r_{r 1} & 0 & 0 \\
0 & 0 & -r_{s 2} & 0 & -r_{g} & 0 \\
0 & 0 & 0 & -r_{r 2} & 0 & -r_{m}
\end{array}\right]
$$

The radii present in matrix $\mathbf{R}$ are defined in Fig. 3 and are constrained as follows: $r_{c}=r_{p}+r_{s 1}$ and $r_{r 1}=2 r_{p}+r_{s 1}$.

\section{A. Direct derivation of the radius matrix $R$.}

Let $r_{i j, i}$ denote the generic coefficient of matrix $\mathbf{R}=$ $\left[r_{i j, i}\right]$ in (5). Subscripts $i j \in\{p s, p r, s g, r m\}$ and $i \in$ $\{c, p, s, r, g, m\}$ indicate that $r_{i j, i}$ is the coefficient that links the tangential force $\vec{F}_{i j}$ to the angular velocity $\vec{\omega}_{i}$. Generalizing the rules provided at the end of Sec. II, one can easily prove that coefficient $r_{i j, i}$ can be expressed as:

$$
r_{i j, i}=S_{F_{i j}} S_{\omega_{i}} r_{i}
$$

where $r_{i}, S_{F_{i j}}$ and $S_{\omega_{i}}$ have the following meaning:

1) $r_{i}$ is the effective radius of angular velocity $\vec{\omega}_{i}$. Two different cases must be considered: a) if velocity $\vec{\omega}_{i}$ a) Direct contact

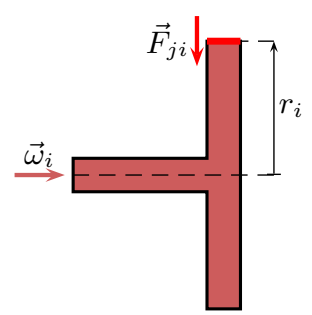

b) Indirect contact

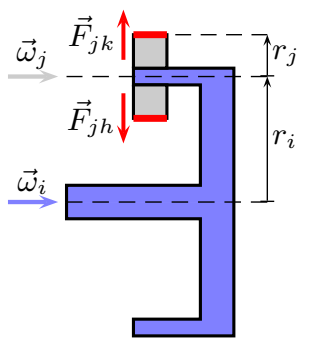

Fig. 5. Effective radius $r_{i}$ : a) Direct contact; b) Indirect contact.

directly affects the force vector $\vec{F}_{i j}$, see Fig. 5.a, then the effective radius $r_{i}$ coincides with the radius of the gear associated with velocity $\vec{\omega}_{i}$; b) if angular velocity $\vec{\omega}_{i}$ affects the force vector $\vec{F}_{i j}$ through an intermediate gear " $j$ ", see Fig. 5.b, then the effective radius $r_{i}$ coincides with the distance between the rotation axes of the two angular velocities $\vec{\omega}_{i}$ and $\vec{\omega}_{j}$.

2) $S_{F_{i j}}$ is the sign of the positive direction of vector $\vec{F}_{i j}$ : $S_{F_{i j}}=\left\{\begin{array}{cl}1 & \text { if } \vec{F}_{i j} \text { is oriented from gear } i \text { to gear } j \\ -1 & \text { if } \vec{F}_{j i} \text { is oriented from gear } j \text { to gear } i\end{array}\right.$

3) $S_{\omega_{i}}$ is related to the sign of the velocity vector $\vec{\omega}_{i}$ :

$$
S_{\omega_{i}}=\left\{\begin{array}{cl}
1 & \text { if } \vec{F}_{i j} \text { is on the left of vector } \vec{\omega}_{i} \\
-1 & \text { if } \vec{F}_{i j} \text { is on the right of vector } \vec{\omega}_{i}
\end{array}\right.
$$

The left and right sides of vector $\vec{\omega}_{i}$ are determined by moving in the positive direction along vector $\vec{\omega}_{i}$.

Applying the previous rules to the gears shown in Fig. 5, it results: a) coefficient $r_{i j, i}$ associated with the direct contact of case a) is $r_{i j, i}=-r_{i}$ because $S_{F_{j i}}=-1$ and $S_{\omega_{i}}=1$; b) coefficients $r_{j k, i}$ and $r_{j h, i}$ associated with the indirect contacts of case b) are $r_{j k, i}=r_{j h, i}=r_{i}$ because $S_{F_{j h}}=$ $S_{F_{j k}}=1$ and $S_{\omega_{i}}=1$.

From (2), it follows that $\dot{x}_{i j}=r_{i j, i} \omega_{i}$ is the tangential velocity of one of the two terminals of spring $K_{i j}$ when the angular velocity $\vec{\omega}_{i}$ moves along its positive direction. Since the sign of velocity $\dot{x}_{i j}$ directly affects the sign of the force vector $\vec{F}_{i j}$, it is evident that $\dot{x}_{i j}$ must change sign both when the velocity vector $\vec{\omega}_{i}$ and when the force vector $\vec{F}_{i j}$ change their positive directions. Finally, Fig. 6 graphically shows why the effective radius of an angular velocity $\vec{\omega}_{i}$ is equal to $r_{i}$ for both case a) and b) of direct and indirect contact. In particular, in the indirect contact case b) the tangential velocity $\dot{x}_{i j}=r_{i} \omega_{i}$ is the same for the two elastic elements $K_{j h}$ and $K_{j k}$ because, when $\vec{\omega}_{i}$ moves along its positive direction, the angular velocity $\vec{\omega}_{j}$ is kept equal to zero.

\section{B. Rigid and reduced model when $\mathbf{K} \rightarrow \infty$.}

When $\mathbf{K} \rightarrow \infty$, from state space model (2) one obtains:

$$
\mathbf{R} \boldsymbol{\omega}=\mathbf{0} \Leftrightarrow\left\{\begin{array}{r}
r_{c} \omega_{c}-r_{p} \omega_{p}-r_{s 1} \omega_{s}=0 \\
r_{c} \omega_{c}+r_{p} \omega_{p}-r_{r 1} \omega_{r}=0 \\
-r_{g} \omega_{g}-r_{s 2} \omega_{s}=0 \\
-r_{m} \omega_{m}-r_{r 2} \omega_{r}=0
\end{array}\right.
$$


a) Direct contact

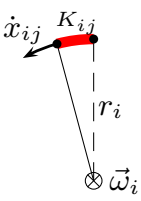

Fig. 6. Angular velocities $\vec{\omega}_{i}$ and tangential velocities $\dot{x}_{i j}$.

System (6) provides four static constraints between the six components $\omega_{i}$ of the angular velocity vector $\boldsymbol{\omega}$, meaning that vector $\boldsymbol{\omega}$ can be expressed as a function of "two" of the six composing angular velocities: $\boldsymbol{\omega}=\mathbf{Q}_{1} \mathbf{x}_{1}$, where $\mathbf{x}_{1}=\left[\begin{array}{ll}\omega_{i} & \omega_{j}\end{array}\right]^{\mathrm{T}}$. By choosing $\mathbf{x}_{1}=\left[\begin{array}{ll}\omega_{c} & \omega_{r}\end{array}\right]^{\mathrm{T}}$, one obtains the following congruent state space transformation:

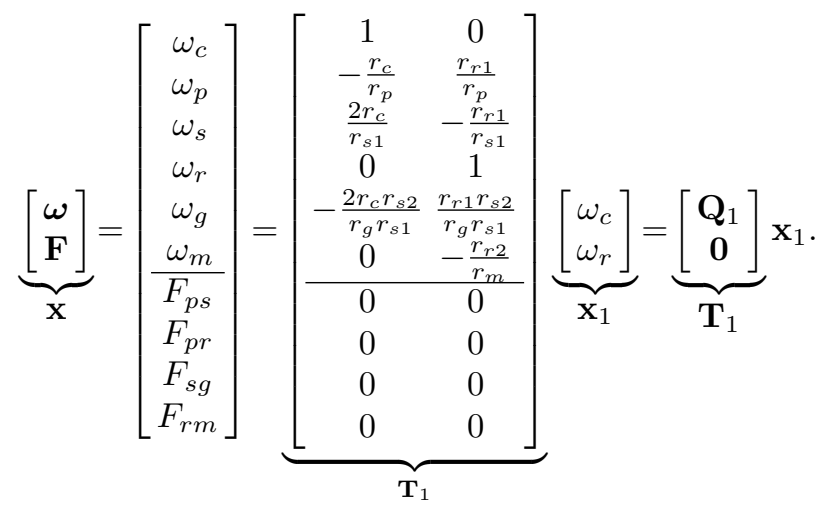

By applying transformation $\mathbf{x}=\mathbf{T}_{1} \mathbf{x}_{1}$ to system (2), one obtains the following reduced system $\mathbf{L}_{1} \dot{\mathbf{x}}_{1}=\mathbf{A}_{1} \mathbf{x}_{1}+\mathbf{B}_{1} \mathbf{u}$ :

$$
\underbrace{\left[\begin{array}{cc}
J_{1} & J_{3} \\
J_{3} & J_{2}
\end{array}\right]}_{\mathbf{L}_{1}} \underbrace{\left[\begin{array}{c}
\dot{\omega}_{c} \\
\dot{\omega}_{r}
\end{array}\right]}_{\dot{\mathbf{x}}_{1}}=\underbrace{\left[\begin{array}{cc}
b_{1} & b_{3} \\
b_{3} & b_{2}
\end{array}\right]}_{\mathbf{A}_{1}} \underbrace{\left[\begin{array}{c}
\omega_{c} \\
\omega_{r}
\end{array}\right]}_{\mathbf{x}_{1}}+\underbrace{\mathbf{Q}_{1}^{\mathrm{T}}}_{\mathbf{B}_{1}} \underbrace{\boldsymbol{\tau}}_{\mathbf{u}}
$$

where martices $\mathbf{L}_{1}, \mathbf{A}_{1}$ and $\mathbf{B}_{1}$ have the following structure:

$$
\left\{\begin{array}{l}
\mathbf{L}_{1}=\mathbf{T}_{1}^{\mathrm{T}} \mathbf{L} \mathbf{T}_{1}=\mathbf{Q}_{1}^{\mathrm{T}} \mathbf{J} \mathbf{Q}_{1} \\
\mathbf{A}_{1}=\mathbf{T}_{1}^{\mathrm{T}} \mathbf{A} \mathbf{T}_{1}=-\underbrace{\mathbf{Q}_{1}^{\mathrm{T}} \mathbf{R}^{\mathrm{T}} \mathbf{B}_{K} \mathbf{R} \mathbf{Q}_{1}}_{\mathbf{0}}-\mathbf{Q}_{1}^{\mathrm{T}} \mathbf{B}_{J} \mathbf{Q}_{1} \\
\mathbf{B}_{1}=\mathbf{T}_{1}^{\mathrm{T}} \mathbf{B}=\mathbf{Q}_{1}^{\mathrm{T}}
\end{array}\right.
$$

Term $\mathbf{Q}_{1}^{\mathrm{T}} \mathbf{R}^{\mathrm{T}} \mathbf{B}_{K} \mathbf{R} \mathbf{Q}_{1}$ is equal to zero because $\mathbf{Q}_{1} \in \operatorname{Ker}(\mathbf{R})$ :

$$
\mathbf{R} \boldsymbol{\omega}=\mathbf{0} \quad \Leftrightarrow \quad \mathbf{R} \mathbf{Q}_{1} \mathbf{x}_{1}=\mathbf{0} \quad \Leftrightarrow \quad \mathbf{R} \mathbf{Q}_{1}=\mathbf{0} .
$$

The structure of elements $J_{1}, J_{3}$ and $J_{2}$ in matrix $\mathbf{L}_{1}$ is:

$$
\begin{aligned}
& J_{1}=J_{c}+\frac{J_{p} r_{c}{ }^{2}}{r_{p}{ }^{2}}+\frac{4 J_{s} r_{c}{ }^{2}}{r_{s 1}{ }^{2}}+\frac{4 J_{g} r_{c}{ }^{2} r_{s 2}{ }^{2}}{r_{g}{ }^{2} r_{s 1}{ }^{2}} \\
& J_{3}=-\frac{J_{p} r_{c} r_{r 1}}{r_{p}{ }^{2}}-\frac{2 J_{s} r_{c} r_{r 1}}{r_{s 1}{ }^{2}}-\frac{2 J_{g} r_{c} r_{r 1} r_{s 2}{ }^{2}}{r_{g}{ }^{2} r_{s 1}{ }^{2}} \\
& J_{2}=J_{r}+\frac{J_{m} r_{r 2}{ }^{2}}{r_{m}{ }^{2}}+\frac{J_{p} r_{r 1}{ }^{2}}{r_{p}{ }^{2}}+\frac{J_{s} r_{r 1}{ }^{2}}{r_{s 1}{ }^{2}}+\frac{J_{g} r_{r 1}{ }^{2} r_{s 2}{ }^{2}}{r_{g}{ }^{2} r_{s 1}{ }^{2}}
\end{aligned}
$$

The structure of elements $b_{1}, b_{3}$ and $b_{2}$ in matrix $\mathbf{A}_{1}$ is:

$$
\begin{aligned}
& b_{1}=-b_{c}-\frac{b_{p} r_{c}^{2}}{r_{p}^{2}}-\frac{4 b_{s} r_{c}^{2}}{r_{s 1}{ }^{2}}-\frac{4 b_{g} r_{c}^{2} r_{s 2}^{2}}{r_{g}{ }^{2} r_{s 1}^{2}} \\
& b_{3}=\frac{b_{p} r_{c} r_{r 1}}{r_{p}^{2}}+\frac{2 b_{s} r_{c} r_{r 1}}{r_{s 1}{ }^{2}}+\frac{2 b_{g} r_{c} r_{r 1} r_{s 2}{ }^{2}}{r_{g}{ }^{2} r_{s 1}{ }^{2}} \\
& b_{2}=-b_{r}-\frac{b_{m} r_{r 2}^{2}}{r_{m}{ }^{2}}-\frac{b_{p} r_{r 1}{ }^{2}}{r_{p}{ }^{2}}-\frac{b_{s} r_{r 1}{ }^{2}}{r_{s 1}{ }^{2}}-\frac{b_{g} r_{r 1}{ }^{2} r_{s 2}{ }^{2}}{r_{g}{ }^{2} r_{s 1}{ }^{2}}
\end{aligned}
$$

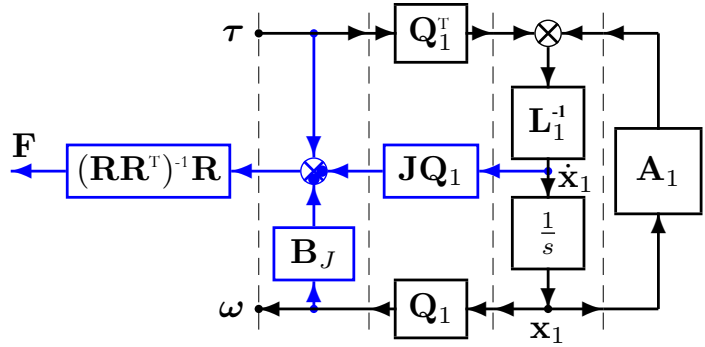

Fig. 7. Modified POG block scheme of the considered planetary gear.

Property 1. The force vector $\mathbf{F}$ is not present in the reduced system (7), but its time behavior can still be obtained using the following relation:

$$
\mathbf{F}=\left(\mathbf{R R}^{T}\right)^{-l} \mathbf{R}\left(\boldsymbol{\tau}-\mathbf{J} \mathbf{Q}_{1} \dot{\mathbf{x}}_{1}-\mathbf{B}_{J} \mathbf{Q}_{1} \mathbf{x}_{1}\right)
$$

Proof. From (2) and (7), one obtains:

$$
\mathbf{R}^{\mathrm{T}} \mathbf{F}=\boldsymbol{\tau}-\mathbf{J} \dot{\boldsymbol{\omega}}-\left(\mathbf{B}_{J}+\mathbf{R}^{\mathrm{T}} \mathbf{B}_{k} \mathbf{R}\right) \boldsymbol{\omega}
$$

When $\mathbf{K} \rightarrow \infty$, it is $\boldsymbol{\omega}=\mathbf{Q}_{1} \mathbf{x}_{1}$. By substituting in the previous relation, it results:

$$
\mathbf{R}^{\mathrm{T}} \mathbf{F}=\boldsymbol{\tau}-\mathbf{J} \mathbf{Q}_{1} \dot{\mathbf{x}}_{1}-\mathbf{B}{ }_{J} \mathbf{Q}_{1} \mathbf{x}_{1}-\mathbf{R}^{\mathrm{T}} \mathbf{B}_{k} \underbrace{\mathbf{R} \mathbf{Q}_{1}}_{\mathbf{0}} \mathbf{x}_{1} .
$$

Relation (8) can be directly obtained from (9) by applying the pseudoinverse of matrix $\mathbf{R}$, that is $\left(\mathbf{R} \mathbf{R}^{\mathrm{T}}\right)^{-1} \mathbf{R}$, on the left of relation (9) and recalling that $\mathbf{R Q}_{1}=\mathbf{0}$.

The modified POG block scheme of Fig. 7 combines in the same figure the computation of the force vector $\mathbf{F}$ (blue blocks) with the POG reduced system (7) (black blocks).

\section{Simulation results}

Both the block scheme reported in Fig. 4, describing the full elastic model, and the one reported in Fig. 7, describing the reduced model, have been simulated in Matlab/Simulink using the following parameters and initial conditions:

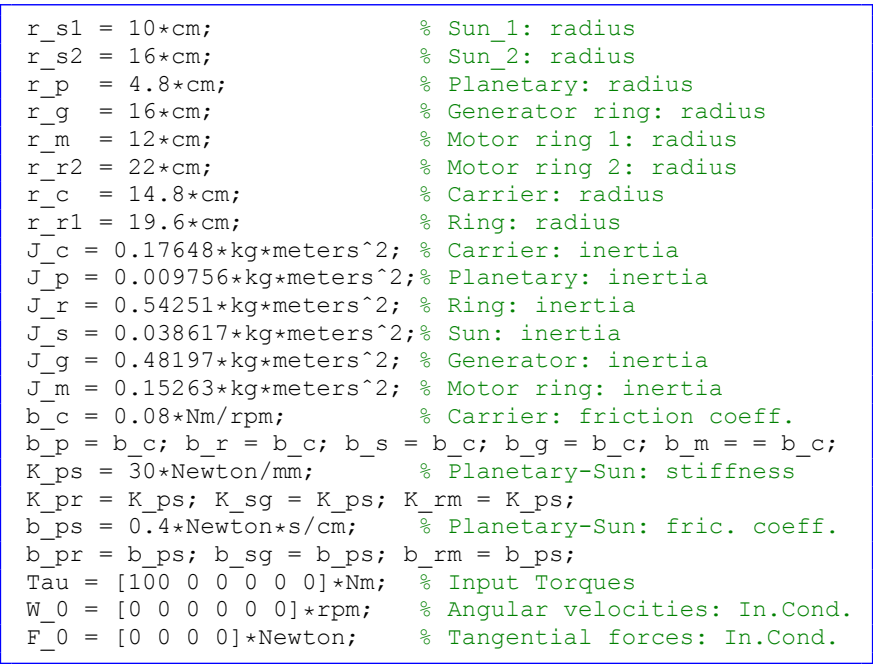

The obtained simulation results are the following: the angular velocities $\omega_{i}$, for $i \in\{c, p, s, r, g, m\}$, are shown in Fig. 8, and the tangential forces $F_{i j}$, for $i j \in\{p s, p r, s g, r m\}$, are shown in Fig. 9. The time behaviors plotted by using dashed 


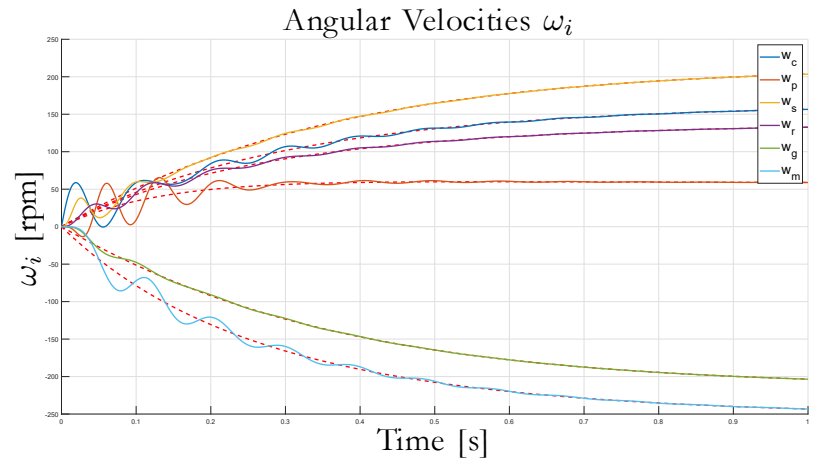

Fig. 8. Angular velocities $\omega_{i}$, for $i \in\{c, p, s, r, g, m\}$.

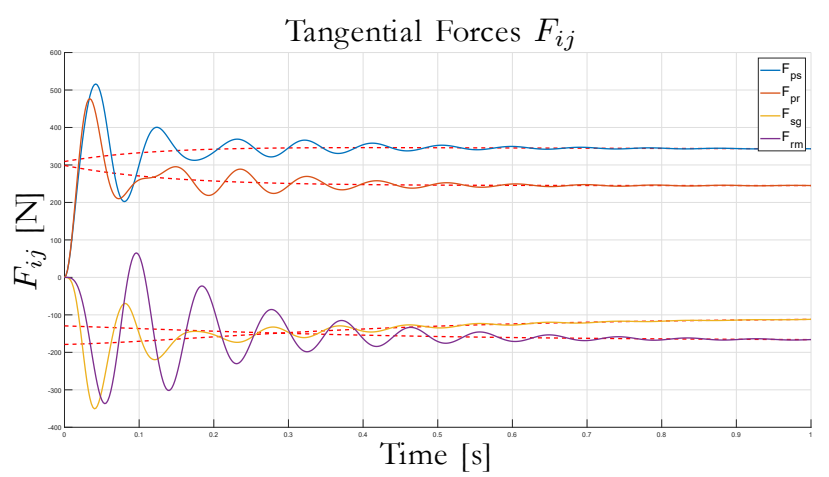

Fig. 9. Tangential forces $F_{i j}$, for $i j \in\{p s, p r, s g, r m\}$.

red lines refer to the simulation results obtained by using the rigid reduced system (7). The time behaviors plotted using colored lines refer to the simulation results obtained by using the extended POG system (2) taking the elasticities into account. From Fig. 8 and 9 it is evident that, after the transient, the time behaviors obtained by using the elastic model (2) asymptotically tend to those obtained by using the reduced model (7). Moreover, the time behaviors of the tangential forces $F_{i j}$ corresponding to the reduced model (7) reported in Fig. 9 have been obtained by means of relation (8), giving a proof of the effectiveness of such relation.

\section{MOdELING A TWO-STAGES PLANETARY GEAR}

Reference is made to the two-stages planetary gear shown in Fig. 10. The extended elastic model of the considered system can be graphically represented by using the POG scheme shown in Fig. 4 once again. The corresponding POG state space model can be obtained by means of the same equations and the same symbols used in (2) and in (3) for the previously considered one-stage planetary gear. As regards the two-stages planetary gear, the velocity vector $\boldsymbol{\omega}$, the input torque vector $\boldsymbol{\tau}$ the force vector $\mathbf{F}$ and the state vector $\mathbf{X}$ are defined as follows:

$$
\boldsymbol{\omega}=\left[\begin{array}{c}
\omega_{s} \\
\omega_{c} \\
\omega_{p} \\
\omega_{a} \\
\omega_{r} \\
\omega_{b} \\
\omega_{q}
\end{array}\right], \quad \boldsymbol{\tau}=\left[\begin{array}{c}
\tau_{s} \\
\tau_{c} \\
\tau_{p} \\
\tau_{a} \\
\tau_{r} \\
\tau_{b} \\
\tau_{q}
\end{array}\right], \quad \mathbf{F}=\left[\begin{array}{c}
F_{s p} \\
F_{s a} \\
F_{p r} \\
F_{a b} \\
F_{b q}
\end{array}\right], \quad \mathbf{x}=\left[\begin{array}{c}
\boldsymbol{\omega} \\
\mathbf{F}
\end{array}\right] .
$$

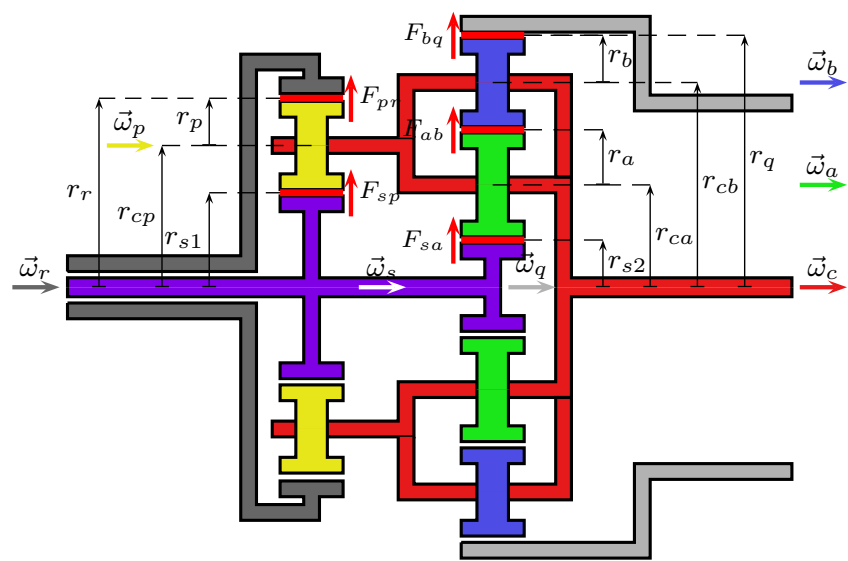

Fig. 10. Basic structure of the considered two-stages planetary gear.

In this case, the inertia and friction matrices $\mathbf{J}$ and $\mathbf{B}_{J}$ are:

$$
\mathbf{J}=\left[\begin{array}{ccccccc}
J_{s} & 0 & 0 & 0 & 0 & 0 & 0 \\
0 & J_{c} & 0 & 0 & 0 & 0 & 0 \\
0 & 0 & J_{p} & 0 & 0 & 0 & 0 \\
0 & 0 & 0 & J_{a} & 0 & 0 & 0 \\
0 & 0 & 0 & 0 & J_{r} & 0 & 0 \\
0 & 0 & 0 & 0 & 0 & J_{b} & 0 \\
0 & 0 & 0 & 0 & 0 & 0 & J_{q}
\end{array}\right], \quad \mathbf{B}_{J}=\left[\begin{array}{ccccccc}
b_{s} & 0 & 0 & 0 & 0 & 0 & 0 \\
0 & b_{c} & 0 & 0 & 0 & 0 & 0 \\
0 & 0 & b_{p} & 0 & 0 & 0 & 0 \\
0 & 0 & 0 & b_{a} & 0 & 0 & 0 \\
0 & 0 & 0 & 0 & b_{r} & 0 & 0 \\
0 & 0 & 0 & 0 & 0 & b_{b} & 0 \\
0 & 0 & 0 & 0 & 0 & 0 & b_{q}
\end{array}\right]
$$

The stiffness and friction matrices $\mathbf{K}$ and $\mathbf{B}_{K}$ are:

$$
\mathbf{K}=\left[\begin{array}{ccccc}
K_{s p} & 0 & 0 & 0 & 0 \\
0 & K_{s a} & 0 & 0 & 0 \\
0 & 0 & K_{p r} & 0 & 0 \\
0 & 0 & 0 & K_{a b} & 0 \\
0 & 0 & 0 & 0 & K_{b q}
\end{array}\right], \mathbf{B}_{K}=\left[\begin{array}{ccccc}
b_{s p} & 0 & 0 & 0 & 0 \\
0 & b_{s a} & 0 & 0 & 0 \\
0 & 0 & b_{p r} & 0 & 0 \\
0 & 0 & 0 & b_{a b} & 0 \\
0 & 0 & 0 & 0 & b_{b q}
\end{array}\right]
$$

As far as the considered two-stages planetary gear is concerned, the radius matrix $\mathbf{R}$ is defined in the following way:

$$
\mathbf{R}=\left[\begin{array}{ccccccc}
r_{s 1} & -r_{c p} & r_{p} & 0 & 0 & 0 & 0 \\
r_{s 2} & -r_{c a} & 0 & r_{a} & 0 & 0 & 0 \\
0 & r_{c p} & r_{p} & 0 & -r_{r} & 0 & 0 \\
0 & r_{c a}-r_{c b} & 0 & r_{a} & 0 & r_{b} & 0 \\
0 & r_{c b} & 0 & 0 & 0 & r_{b} & -r_{q}
\end{array}\right]
$$

Radii $r_{i}$ or $r_{i j}$ present in matrix $\mathbf{R}$ are graphically defined in Fig. 10 and are constrained as follows:

$$
\begin{aligned}
r_{c p}=r_{p}+r_{s 1}, & r_{c b}=2 r_{a}+r_{b}+r_{s 2}, \\
r_{c a}=r_{a}+r_{s 2}, & r_{q}=2 r_{a}+2 r_{b}+r_{s 2} .
\end{aligned}
$$

The radii within matrix $\mathbf{R}$ defined in (10) have been computed by means of the rules defined in Sec. III-A. As an example, let us refer to element $r_{a b, c}$ which falls within the indirect contact case (see Fig. 5.b and 6.b). This represents a particular case, as the angular velocity $\vec{\omega}_{c}$ affects the force vector $\vec{F}_{a b}$ through two different intermediate gears, characterized by moments of inertia $J_{a}$ and $J_{b}$. It follows that $r_{a b, c}$ is given by the linear combination of two radii:

- $\vec{\omega}_{c}$ affects $\vec{F}_{a b}$ through $J_{a}$, therefore it is $S_{F_{a b}}=1$ and $S_{\omega_{c}}=1$, meaning that $r_{a b, c}^{\prime}=r_{c a}$; 
- $\vec{\omega}_{c}$ affects $\vec{F}_{a b}$ through $J_{b}$, therefore it is $S_{F_{a b}}=-1$ and $S_{\omega_{c}}=1$, meaning that $r_{a b, c}^{\prime \prime}=-r_{c b}$;

From which the overall radius $r_{a b, c}$ can be computed:

$$
r_{a b, c}=r_{a b, c}^{\prime}+r_{a b, c}^{\prime \prime}=r_{c a}-r_{c b} .
$$

When $\mathbf{K} \rightarrow \infty$, see (2), the angular velocities satisfy relation $\mathbf{R} \boldsymbol{\omega}=\mathbf{0}$ and are constrained as follows:

$$
\left\{\begin{array}{r}
r_{p} \omega_{p}-r_{c p} \omega_{c}+r_{s 1} \omega_{s}=0 \\
r_{a} \omega_{a}-r_{c a} \omega_{c}+r_{s 2} \omega_{s}=0 \\
r_{c p} \omega_{c}+r_{p} \omega_{p}-r_{r} \omega_{r}=0 \\
r_{a} \omega_{a}+r_{b} \omega_{b}+\omega_{c}\left(r_{c a}-r_{c b}\right)=0 \\
r_{b} \omega_{b}+r_{c b} \omega_{c}-r_{q} \omega_{q}=0
\end{array}\right.
$$

In this case, by choosing $\mathbf{x}_{1}=\left[\omega_{c} \omega_{r}\right]^{\mathrm{T}}$, from (11) one obtains the congruent state space transformation $\mathbf{x}=\mathbf{T}_{1} \mathbf{x}_{1}$ where $\mathbf{T}_{1}=\left[\begin{array}{c}\mathbf{Q}_{1} \\ \mathbf{0}\end{array}\right] \mathbf{x}_{1}$ and matrix $\mathbf{Q}_{1}$ is defined as follows:

$$
\mathbf{Q}_{1}=\left[\begin{array}{cc}
1 & 0 \\
\frac{r_{s 1}}{2 r_{p p}} & \frac{r_{r}}{2 r_{c p}} \\
-\frac{r_{s 1}}{2 r_{p}} & \frac{r}{2 r_{p}} \\
\frac{r_{c a} r_{s 1}-2 r_{c p} r_{s 2}}{2 r_{a} r_{c p}} & \frac{r_{c a} r_{r}}{2 r_{a} r_{c p}} \\
0 & 1 \\
\frac{r_{c b} r_{s 1}-2 r_{c a} r_{s 1}+2 r_{c p} r_{s 2}}{2 r_{b} r_{c p}} & -\frac{2 r_{c a} r_{r}-r_{c b} r_{r}}{2 r_{b} r_{c p}} \\
\frac{r_{c b} r_{s 1}-r_{c a} r_{s 1}+r_{c p} r_{s 2}}{r_{c p} r_{q}} & -\frac{r_{c a} r_{r}-r_{c b} r_{r}}{r_{c p} r_{q}}
\end{array}\right]
$$

By using the congruent transformation $\mathbf{x}=\mathbf{T}_{1} \mathbf{x}_{1}$ to system (2), one obtains a reduced system having the same structure of the reduced model (7) obtained in the previous example. In this case, the analytical expressions of $J_{1}, J_{3}$ and $J_{2}$ in matrix $\mathbf{L}_{1}$, together with the analytical expressions of $b_{1}, b_{3}$ and $b_{2}$ in matrix $\mathbf{A}_{1}$, are not reported in this paper because they are too long. Even in this case, the force vector $\mathbf{F}$ can be recovered from the reduced POG model (7) by using (8).

\section{A. Simulation results}

The two-stages planetary gear has been simulated in Matlab/Simulink by using the following parameters:

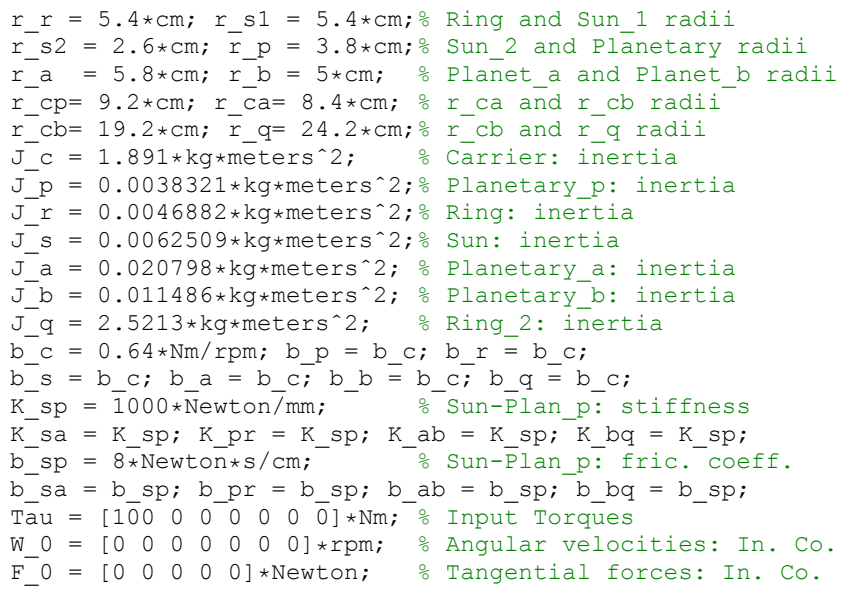

The angular velocities $\omega_{i}$, for $i \in\{c, p, s, r, g, m\}$, are shown in Fig. 11; the tangential forces $F_{i j}$, for $i j \in$ $\{p s, p r, s g, r m\}$, are shown in Fig. 12.

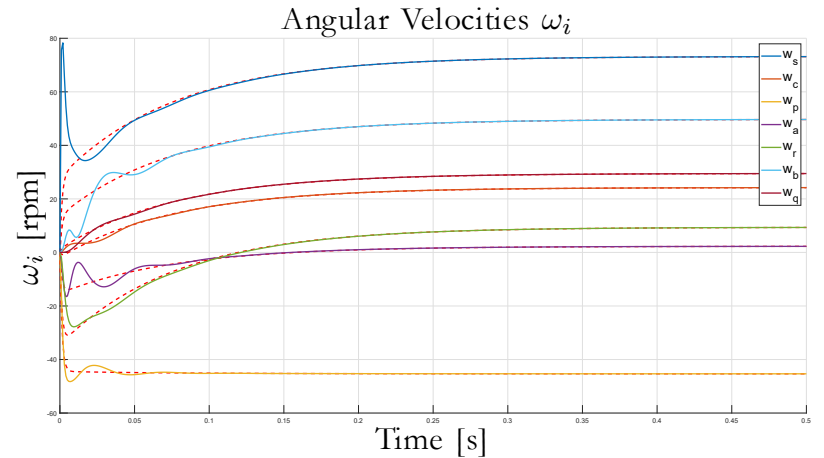

Fig. 11. Angular velocities $\omega_{i}$, for $i \in\{s, c, p, a, r, b, q\}$.

Tangential Forces $F_{i j}$

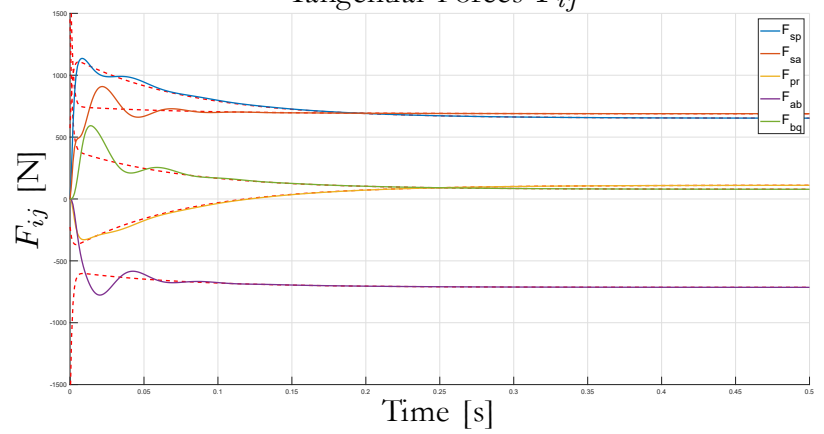

Fig. 12. Tangential forces $F_{i j}$, for $i j \in\{s p, s a, p r, a b, b q\}$.

\section{CONCLUSIONS}

In this paper, a new method for modeling planetary gear transmission systems has been presented. The method provides the user with both the full elastic model of the system and the reduced rigid one. The POG modeling technique allows the user to select the angular speeds to be kept as state variables in the reduced model. Moreover, the reduced model also provides the tangential elastic forces present between the gears. The presented modeling method has been tested on two different planetary gear systems, which have been modeled and simulated in Matlab/Simulink. The simulative results have been reported in this paper and show the effectiveness of the presented method.

\section{REFERENCES}

[1] A. Mohsine, E. M. Boudi, A. E. Marjani, "Modeling and Structural Analysis of Planetary Gear of a Wind Turbine", International Renewable and Sustainable Energy Conference (IRSEC), Marrakech, Morocco, November 14-17, 2016.

[2] H. Jingnan, W. Shaohong, M. Chao, "Nonlinear dynamic analysis of $2 \mathrm{~K}-\mathrm{H}$ planetary gear transmission system", 13th IEEE International Conference on Electronic Measurement \& Instruments (ICEMI), Yangzhou, China, October 20-22, 2017.

[3] Q. Tao, J. Zhou, W. Sun, J. Kang, "Study on the Inherent Characteristics of Planetary Gear Transmissions", 21st International Conference on Automation and Computing (ICAC), Glasgow, UK, Sept. 11-12, 2015.

[4] J. Liu, H. Peng, "Modeling and Control of a Power-Split Hybric Vehicle", IEEE Trans. on Control Systems Technology, Nov. 2008.

[5] X. Zhang, H. Peng, J. Sun, S. Li, "Automated Modeling and Mode Screening for Exhaustive Search of Double-Planetary-Gear Power Split Hybrid Powertrains", ASME Dynamic Systems and Control Conference, San Antonio, Texas, Oct 22-24, 2014.

[6] R. Zanasi, "The Power-Oriented Graphs Technique: System modeling and basic properties", IEEE Vehicle Power and Propulsion Conference, Lille, France, Sept. 1-3, 2010. 